\title{
TRAFIC: Statistical Plan for a Pragmatic Early Phase 1/2 Bayesian Adaptive Dose Escalation Trial in Rheumatoid Arthritis
}

\section{Michael Cole}

Newcastle University School of Population and Health Sciences: Newcastle University Institute for Health and Society

\section{Christina Yap}

Institute of Cancer Research: The Institute of Cancer Research

\section{Christopher Buckley}

University of Birmingham

Wan-Fai $\mathrm{Ng}$

Newcastle University Institute of Cellular Medicine

\section{lain Mclnnes}

University of Glasgow

\section{Andrew Filer}

University of Birmingham College of Medical and Dental Sciences

\section{Stefan Siebert}

University of Glasgow

\section{Arthur Pratt}

Newcastle University Faculty of Medical Sciences

John D Isaacs

Newcastle University Institute of Cellular Medicine

Deborah D Stocken ( $\nabla$ d.d.stocken@leeds.ac.uk)

University of Leeds https://orcid.org/0000-0001-8031-1738

\section{Research Article}

Keywords: CRM, dose-finding, adaptive, early-phase, clinical trial

Posted Date: April 13th, 2021

DOI: https://doi.org/10.21203/rs.3.rs-340897/v1

License: (c) (1) This work is licensed under a Creative Commons Attribution 4.0 International License. 
Version of Record: A version of this preprint was published at Trials on July 6th, 2021. See the published version at https://doi.org/10.1186/s13063-021-05384-5. 
TRAFIC: Statistical Plan for a Pragmatic Early Phase 1/2 Bayesian Adaptive Dose Escalation Trial in Rheumatoid Arthritis

\author{
M Cole ${ }^{1}$, C Yap ${ }^{2}$, Buckley $C^{3}$, WF Ng ${ }^{4}$, I Mclnnes $^{5}$, A Filer ${ }^{3}$, S Siebert ${ }^{5}$, A Pratt ${ }^{4}, J D$ \\ Isaacs $^{4}$, DD Stocken ${ }^{6}$ \\ 1. Institute of Health and Society, Newcastle University. Email: \\ michael.cole@newcastle.ac.uk
}

2. Clinical Trials and Statistics Unit, The Institute of Cancer Research, London Email: christina.yap@icr.ac.uk

3. School of Immunity and Infection, University of Birmingham Email:

\title{
c.d.buckley@bham.ac.uk; a.filer@bham.ac.uk
}

4. Institute of Cellular Medicine, Newcastle University Email: wan-fai.ng@ncl.ac.uk; arthur.pratt@ncl.ac.uk; john.isaacs@ncl.ac.uk

5. College of Medical, Veterinary and Life Sciences, University of Glasgow Email: iain.mcinnes@glasgow.ac.uk; Stefan.siebert@glasgow.ac.uk

6. Leeds Institute of Clinical Trials Research, University of Leeds. Email: d.d.stocken@leeds.ac.uk

Correspondence to:

Professor Deborah D Stocken PhD CStat, Leeds Institute of Clinical Trials Research University of Leeds, d.d.stocken@leeds.ac.uk 


\section{ABSTRACT}

Background: Adaptive model-based dose-finding designs have demonstrated advantages over traditional rule-based designs but have increased statistical complexity resulting in slow uptake especially outside of cancer trials. TRAFIC is a multi-centre, early phase trial in Rheumatoid Arthritis incorporating a model-based design.

Methods: A Bayesian adaptive dose-finding phase I trial rolling into a single arm, single stage phase II trial. Model parameters for phase I were chosen via Monte Carlo simulation evaluating objective performance measures under clinically relevant scenarios and incorporated stopping rules for early termination. Potential designs were further calibrated utilising dose transition pathways.

Discussion: TRAFIC is an MRC funded trial of a re-purposed treatment demonstrating that it is possible to design, fund and implement a model-based phase I trial in a noncancer population within conventional research funding tracks and regulatory constraints. The phase I design allows borrowing of information from previous trials; all accumulated data to be utilised in decision-making; verification of operating characteristics through simulation; improved understanding for management and oversight teams through dose transition pathways. The rolling phase II design brings efficiencies in trial conduct including site and monitoring activities, and cost. TRAFIC is the first funded model-based dose-finding trial in inflammatory disease demonstrating that small phase I/II trials can have an underlying statistical basis for decision-making and interpretation. 
Keywords: CRM, dose-finding, adaptive, early-phase, clinical trial Trial Registration: ISRCTN 36667085

\section{BACKGROUND}

Development of novel therapies especially in the cancer arena, has brought efficient, contemporary statistical model-based dose-finding designs to assess levels of toxicity and activity. Transfer of such approaches to other diseases is warranted. Adaptive model-based designs for dose-finding studies are based on making dose recommendations given all accumulated data at that time. They have demonstrated advantages to traditional rule based designs, such as the $3+3$ design ${ }^{1,2}$ but have increased statistical complexity resulting in slow uptake. Adoption is improving within the cancer clinical trial setting but remains poor - only $1.6 \%$ of trials published 1991 to 2006 used model-based approaches ${ }^{3}$ increasing to only $6.4 \%$ of trials published 2012 to $2014^{4}$.

Rheumatoid arthritis (RA) afflicts $0.5-1.0 \%$ of adults globally. It presents polyarthritis with coincident morbidities including vascular bone and cognitive deficits and together these substantially impact quality of life, disability and long-term survival. Around a third of patients have stopped working within two years of onset and around a half by ten years with significant costs to the economy ${ }^{5}$. Advances in RA management and biologic therapies have contributed to an improved prognosis but up to $50 \%$ fail to achieve remission, with $5-10 \%$ being refractory to all treatments. Consequently, there remains a significant unmet need in 
RA management requiring novel therapeutic approaches. Prior therapeutics have focussed primarily on immune based therapeutics. We sought to evaluate a stromal targeting strategy and since this is an entirely novel approach we required alternative methods to minimise patient exposure pending estimation of safety and initial indication of potential efficacy.

There is a paucity of early phase adaptive designs across all fields of medicine. A systematic review in rheumatology ${ }^{6}$ identified just one adaptive early phase design from 62 trials considered. In this paper we report the statistical design, calibration and implementation of the Targeting the Rheumatoid Arthritis Synovial Fibroblast with Cyclin-Dependent Kinase Inhibition (TRAFIC) trial. TRAFIC is a non-commercial, multi-centre, phase I/II trial incorporating a Bayesian adaptive model-based dose finding phase I design to determine the safety, tolerability and efficacy of seliciclib as an addition to existing therapy in patients with RA. Rolling the trial from phase I to phase II allows site activity and momentum to be retained bringing trial conduct efficiencies including internal and external trial monitoring activities, and associated cost savings. It also allows continuity for clinical, trial and monitoring teams. These advantages, and also potential advantages of 'sharing' patients from one phase to the next, make this approach attractive when conducting challenging early phase trials.

Seliciclib (R-roscovitine) is an orally available cyclin dependent kinase inhibitor with an acceptable toxicity profile ${ }^{7}$ repurposed from the oncology setting. Determining the toxicity profile of seliciclib used in combination with a biologic plus or minus conventional synthetic 
disease-modifying anti-rheumatic drugs (sDMARDs) is an essential component of TRAFIC, providing important insight into its potential acceptability as an adjunctive therapy in RA.

\section{METHODS}

\section{Statistical Design}

TRAFIC has a phase I dose finding trial rolling into a single arm, single stage phase II trial. The primary objective of phase I is to determine the maximum tolerated dose (MTD) of seliciclib over a 4 week treatment period when given in addition to an existing TNF inhibitor with or without sDMARDs. Phase I is planned to roll into phase II for which the primary objective is to assess the potential efficacy of seliciclib following 12 weeks of treatment when administered at the MTD established in phase I; efficacy is based on a composite response measure.

\section{Phase I Continual Reassessment Method Dose Finding Design}

The MTD will be established using a modified one-stage Bayesian Continual Reassessment Method (CRM) model-based phase I design ${ }^{8}$. The primary outcome is identification of MTD based on the occurrence of dose limiting toxicities (DLTs). A DLT is defined as the cessation of the Investigational Medicinal Product (IMP) due to adverse events or reactions (AE/AR) occurring during the four week treatment period. These can be either symptomatic (e.g. nausea) or abnormal laboratory parameters or investigations. A DLT may be based upon the patient's request to stop treatment, regardless of severity, or an abnormal laboratory parameter necessitating cessation of treatment. In the event of several AEs/ARs contributing 
to the decision to discontinue IMP, only a single DLT will be recorded for the purposes of dose pathway decision making (see below). A flare of RA will not be considered an AE. The primary objective is to identify the MTD associated with a target 0.35 DLT probability. The definition of a DLT and its target probability was agreed at a TRAFIC Investigators consensus meeting. The MTD is defined as the dose level that is closest to the level at which $35 \%$ of patients experience a DLT over the treatment period (4 weeks) of seliciclib, comparable to the rate of sustained tolerated treatment on conventional synthetic sDMARDs such as Methotrexate ${ }^{9}$.

Phase I will include a maximum of 21 patients. Up to seven cohorts of three participants each will be treated. The decision to recruit three patients per cohort was logistical and with respect to the proposed 35\% DLT rate. The number of cohorts was based on the selected dose range which encompasses up to five doses of IMP: participants will receive either $200 \mathrm{mg}, 400 \mathrm{mg}, 600 \mathrm{mg}, 800 \mathrm{mg}$ or $1000 \mathrm{mg}$ seliciclib daily for 4 consecutive days (one cycle) every week for 4 weeks. The dose range and schedule is based on healthy control data and oncology experience ${ }^{7}$. The prior estimate of MTD is $600 \mathrm{mg}$ (Dose Level 3) of seliciclib (Table 1) but, to exercise caution, $400 \mathrm{mg}$ seliciclib (Dose Level 2 ) is defined as the starting dose. The design allows for de-escalation to a lower dose of 200mg (Dose Level 1). Initial estimates of the probability of DLT at each of the dose levels (known as the skeleton) were chosen via Monte Carlo simulation evaluating objective performance measures under clinically relevant scenarios and incorporated stopping rules for early termination. 
The recommended dose (the dose with estimated DLT probability closest to the target of $35 \%$ ) for each of the subsequent cohorts is determined using the CRM incorporating all of the accumulated DLT outcomes but for added safety the design includes a restriction to prevent skipping of untested doses when escalating. Recruitment continues until either the maximum sample size is reached, the trial is stopped early due to unacceptable levels of DLT at the lowest dose or the MTD is considered sufficiently well estimated. Two early stopping rules are included to allow for early termination:

1. If there is a high probability $(>0.7)$ that the posterior probability of DLT at the lowest dose is greater than the target DLT rate of $35 \%$, indicating that the lowest dose is too toxic.

2. If four consecutive cohorts (three patients in each cohort) have already been allocated at the current MTD, which would also be the recommended dose level for the next cohort if the trial continued.

The value of 0.7 was selected so that the design will recommend stopping early for excessive toxicity when we observe 2 or 3 DLTs out of the first 3 patients at the lowest dose (200mg) (Figure 1 - Dose transition pathways flow diagram for first three cohorts).

Parameters and model specification were agreed upfront and inform the most relevant statistical design ${ }^{10}$. Dose transition pathways (DTP) are utilised i) as a calibration tool to help evaluate potential designs and ii) to visualise projected dose recommendations for clinical acceptance ${ }^{11,12}$. In addition, the performance of the proposed design was evaluated through simulation. The DTP allows pathways to be projected for all subsequent cohorts, allowing 
recruitment to proceed if the outcome of the final patient within the current cohort does not alter the dose recommendation for the next cohort. However, for TRAFIC, to avoid potential bias at trial conduct, only the next cohort is displayed. If implemented in full, this strategy can reduce the operational demands on the trial management group, including the trial statistician. Decisions to progress to the next cohort, are considered by the TRAFIC Data Monitoring Committee (DMC) who make recommendations regarding the continuation of the trial.

At trial completion, the number of patients experiencing DLT at each dose level, together with the proportion of patients with DLT at that dose level, will be reported. The Bayesian posterior probability of DLT at each dose level (with $90 \%$ probability interval) will be reported graphically and in tabular form. The MTD will be reported as well as the posterior probability that the DLT rate at dose level $1(200 \mathrm{mg})$ is greater than the target level of $35 \%$. Secondary outcome measures, including PD biomarkers and PK parameters, will be presented descriptively and graphically.

\section{CRM Model Details}

The one-stage, one parameter Bayesian logistic dose-toxicity model used in TRAFIC is given by:

$$
\vartheta(x, \beta)=\frac{\exp \left(3+\mathrm{e}^{\beta} x\right)}{1+\exp \left(3+\mathrm{e}^{\beta} x\right)} \quad \text { for }-\infty<x<\infty \text {, }
$$


where $x$ is the scaled dose and $\vartheta(x, \beta)$ is the probability of a DLT at dose $x(10)$. The model parameter $\beta$ is assumed to be random with prior distribution $\mathrm{N}\left(0, \sigma_{\beta}^{2}\right)$. The Bayesian CRM model is completed by specification of the prior probability of a DLT associated with the five test doses (the skeleton) and $\sigma_{\beta}^{2}$, the prior variance of $\beta$.

Early termination is allowed if there is a high probability that the posterior probability of DLT at the lowest dose is greater than the target DLT rate. The posterior distribution of the DLT occurrence probability at the lowest dose requires the posterior distribution for $\beta$ to be evaluated. After the first $n$ patients, this is given by:

$p\left(\beta \mid X_{n}, Y_{n}\right) \propto \exp \left(\frac{-\beta^{2}}{2 \sigma_{\beta}^{2}}\right) \prod_{i=1}^{n} \vartheta\left(x_{i}, \beta\right)^{y_{i}}\left(1-\vartheta\left(x_{i}, \beta\right)\right)^{1-y_{i}}$

where $X_{n}=\left\{x_{1}, x_{2}, \ldots, x_{n}\right\}$ are the scaled doses and $Y_{n}=\left\{y_{1}, y_{2}, \ldots, y_{n}\right\}$ are the DLT outcomes (0 or 1$)$.

The posterior probability that the DLT occurrence probability at Dose Level 1 is greater than some rate $\vartheta_{L}$ is given by $\int_{-\infty}^{\beta_{L}} p\left(\beta \mid X_{n}, Y_{n}\right) d \beta$ where $\beta_{L}=\log \left\{\frac{\log \left(\frac{\vartheta_{L}}{1-\vartheta_{L}}\right)-3}{x_{D 1}}\right\}$ and $x_{D 1}$ is the scaled dose for Dose Level 1. The integral $\int_{-\infty}^{\beta_{L}} p\left(\beta \mid X_{n}, Y_{n}\right) d \beta$ is analytically intractable and so is obtained by numerical integration (13).

The Bayesian CRM model is calibrated (specification of the skeleton and prior variance of $\beta$ ) using the following algorithm of Cheung ${ }^{10}$ which was implemented using a modified version of the $\mathrm{R}$ function mtrials part of the $d f c r m$ R library ${ }^{14}$. 
a) For a given indifference interval half-width $\delta$, the associated skeleton is obtained using the $d f c r m$ function getprior. The indifference interval is an interval into which the DLT probability of the selected dose will eventually fall given a sufficiently large sample size.

b) For each $\delta$, the least informative prior standard deviation for the slope parameter $\beta$, $\sigma_{\beta}^{\mathrm{LI}}(\delta)$, is determined. The SD is defined to be least informative if the prior probability that a dose level is the MTD is approximately equal for all dose levels. Table 1 shows the effect of varying $\sigma_{\beta}$ for TRAFIC when $\delta=0.06$; a large $\sigma_{\beta}$ does not necessary correspond to an uninformative prior for the MTD dose level.

c) The pair $\left\{\delta, \sigma^{\mathrm{LI}} \beta(\delta)\right\}$ was chosen based upon evaluation of the following performance measures: i) the ability to correctly select the true MTD - the risk-adjusted average accuracy, $A_{N}$ (weighting the probability of selecting a dose level by the absolute discrepancy between the true probability of toxicity at that dose level and the target probability of toxicity), and the unadjusted probability of correctly selecting the true MTD; ii) optimal allocation defined as the mean proportion of patients treated within one dose level of the true MTD; iii) the mean proportion of patients treated at an overdose (a dose above the true MTD); and iv) the mean number of patients treated ${ }^{10}$. 


\section{Simulations}

The performance measures in c) above were estimated by Monte Carlo simulation: 20,000 trials were simulated for different values of $\delta$ (0.02 to 0.20 in steps of 0.01$)$. Trials were simulated assuming the underlying probabilities of DLT (Table 2), these are the appropriate plateau calibration configurations with five test dose levels when the target DLT rate is $35 \%$. The performance of the design also was assessed via simulation under several clinically relevant scenarios.

Dose level 1 (200mg) was thought unlikely to be efficacious, hence calibration of the CRM focused on achieving: 1) good accuracy, optimal allocation and minimising the proportion of patients treated at an overdose when the true simulated MTD was at dose levels 2 to 5 ; and 2) minimising the number of patients treated when the true MTD was at dose level 1.

Performance measures implementing the early stopping rules 1 and 2 are plotted against $\delta$ (Figure 2) for each of the calibration curves (Table 2).

The mean of the performance measures taken over the calibration curves $2,3,4$ and 5 , i.e. when the true simulated MTD is at dose levels 2, 3, 4 and 5 are given in Table 3 and Figure 3. The performance measures: 1) $A_{N} ; 2$ ) the unadjusted probability of selecting the true MTD; 3) the mean proportion of patients treated at an overdose; and 4) the mean proportion of patients treated within 1 dose of the true MTD, all show a similar relationship with $\delta$. As $\delta$ increases, the measures remain fairly constant until $\delta=0.06$ when they drop significantly. Except at very low levels of $\delta$, the mean number of patients treated 
decreases as $\delta$ increases, particularly so when the simulated true MTD is dose level 1. The variation in $A_{N}$ over the calibration curves 2, 3, 4 and 5, SD $\left(A_{N}\right)$, is smallest when $\delta=0.04$ and remains low until $\delta$ exceeds 0.06 when it increases rapidly. This variation should be low to ensure reasonable performance across dose levels 2 to 5 , as opposed to good performance at some doses and poor performance at others. Given the objective of achieving a balance between good performance when the true MTD is at dose levels 2 to 5 and minimising the number of patients treated when the true MTD is at dose level 1 , $\delta=0.06$ was selected .

Simulations to assess the chosen level of $\delta$ for various dose-toxicity relationships (Table 4) assume both the true probability of DLT follows the skeleton chosen for TRAFIC (Simulation 1) and where the assumed true MTD across the five dose levels is varied (Simulations 2-6). The accuracy index $\mathscr{A}_{\mathbb{N}}$ is above 0.90 for each simulation. The final design parameters are summarised in Table 5.

\section{Phase II Fleming A'hern Design}

Phase II is a single arm, single stage early phase trial based on a Fleming-A'Herns design ${ }^{15}$ recruiting a total of 18 patients at the recommended phase II dose who provided written informed consent, with baseline and 12-week outcome data. Efficacy is assessed via clinical assessments, analysis of synovial biopsies, and contrast enhanced MRI of an affected hand and wrist. The primary outcome measure is a composite response rate at 12 weeks defined as achieving two of the following three criteria: i) EULAR moderate response or ACR20 
response, ii) histological reduction in macrophage number in the sub lining layer of the synovium $\geq 20 \%$ and iii) reduction of Rheumatoid Arthritis MRI Scoring System score, on MRI, of $\geq 0.5$ units or osteitis score of $\geq 0.2$ units. Secondary outcome measures are as for phase I and, additionally, changes in pharmacodynamic biomarkers in synovial tissue and response rate after 1, 2, 3, 6 and 9 weeks of therapy.

Response rate will be calculated as the total number of patients responding as a proportion of all patients who start treatment. Any patients who are not assessable at 12 weeks will be classed as a non-responder. Individual components of the composite response outcome will be reported descriptively. Adverse events will be reported as the number of patients experiencing an event as a proportion of the total number of patients starting treatment, reported descriptively. Pharmacokinetic (PK) parameters and pharmacodynamic (PD) biomarkers will be presented graphically.

The Fleming A'Hern design assumes a composite response rate to reject Seliciclib (p0) $<25 \%$ and a response rate to investigate Seliciclib further $(p 1)>50 \%$. The justification to investigate Seliciclib further is based on observing a critical minimum number of responses, as specified in the Statistical Analysis Plan. As an early phase trial the error levels are inflated but restricted to an acceptable level of $<15 \% \alpha$ (type 1 ) and $<20 \% \beta$ (type 2 ). With these stated parameters the target recruitment for phase II is calculated as 18 patients. 
At the conclusion of phase II, the Fleming A'Hern design would indicate no further investigation is warranted if the observed number of clinical responses is less than the critical number, retained in the statistical trial master file. As this is the first trial investigating seliciclib in this indication (as a repurposed drug with a novel mechanism of action), the decision to continue will also be based on PD biomarkers and PK parameters, since the TRAFIC Trial Management Group would not want to reject a potentially active drug which has not achieved pragmatic clinical measures of efficacy. In terms of synovial PD biomarkers, the decision to continue will be based upon the following observations and evidence: i) Taqman low density transcriptional arrays incorporating genes of interest relevant to fibroblast biology, inflammation, cell cycle and apoptosis, applied to mRNA extracted from whole synovial tissue; ii) PD effects of seliciclib on the synovial fibroblast; and iii) markers of cell proliferation, such as Ki-67.

\section{DISCUSSION}

The TRAFIC trial is the first reported dose finding trial using a model-based design in inflammatory disease. It is a non-commercial, multi-centre, phase I/ II early phase Bayesian adaptive model-based dose finding trial designed to determine the safety, tolerability and efficacy of seliciclib as an addition to existing therapy in patients with RA. . There remains a clinical need for novel therapeutics or drug repurposing in rheumatoid arthritis given suboptimal responses to all available therapies in a significant proportion of patients. Moreover the field needs to be able to make rapid decisions with new modes of action as early as possible to optimise trial recruitment. Efficient designs are required to minimise patient 
exposure pending estimation of safety. TRAFIC is a rolling phase I/ II trial bringing efficiencies in site activity, patient numbers, external monitoring and costs, minimising some of the challenges when conducting early phase trials.

There are demonstrated advantages of model-based designs for determining maximum tolerated dose in dose finding studies but uptake is slow in disciplines outside of cancer trials. The statistical calibration and implementation of the TRAFIC trial demonstrate that it is possible to design, fund and implement a model-based Continual Reassessment Method phase I trial in a non-cancer population within research funding and regulatory constraints. The simulation approach extends the work of Cheung ${ }^{10}$ by incorporating the two stopping rules used in TRAFIC.

The advantages of the Bayesian approach allow shared learning of information from cancer patients to inform the TRAFIC trial design parameters. The Continual Reassessment Method allows all available accumulated data to be utilised to inform decision making regarding dose escalation, de-escalation or retention through progressive cohorts. Simulation allows the operating characteristics of the proposed adaptive design to be assessed. At the design stage, the use of the Dose Transition Pathways (DTP) encourages closer engagement with the clinical investigators, trials management team and statisticians and improves understanding of how such a model may work in practice. Such discussions are particularly helpful to gather and incorporate vital clinical opinions in the development and calibration of the model. Both simulations and DTP play an instrumental role in the successful 
implementation of this method, which is considerably more complex than a simple rule-based design such as the $3+3^{2,11}$. The phase II design of TRAFIC demonstrates that even small, single arm trials can have an underlying statistical basis for decision making and interpretation, often discounted.

Operationally, identified barriers including longer set-up time for statistical simulation and trial management implementation are beginning to be addressed through NIHR and UKCRC training for both clinical and statistical teams alike ${ }^{16}$. The TRAFIC trial design was supported through NIHR UKCRC CTU infrastructure funding prior to protocol development being funded by MRC. There still remains a need for unified support from regulators and journal editors to promote more accurate dosing. Implementation of a Dose Transition Pathway allows a transparent, graphical interpretation of the trial design to facilitate upfront discussion and decision-making with clinical teams. It can enable an efficient look ahead strategy although any associate bias in doing so must be considered. The Dose Transition Pathway can provide understanding and guidance to the Data Monitoring Committee and is recommended to facilitate upfront discussion and consensus on trial conduct ${ }^{11}$.

The rolling design recruiting patients seamlessly from phase I to phase II allows site activity and momentum to be retained and also brings trial conduct efficiencies including internal site monitoring and external data monitoring activities, with obvious cost savings. The rolling design allows continuity for clinical, trial and monitoring teams maintaining relationships and consistent high quality across trial management activities across phases. A rolling design has 
the potential advantage to 'share' patients from one phase to the next, dependent upon the outcome of the phase I MTD and dose in the final cohort. Altogether, this approach to the trial design is attractive to clinical, trial and site staff and efficient in patient recruitment when conducting challenging early phase trials. The TRAFIC trial demonstrates that small phase I/ II trials can have an underlying statistical basis for decision-making and interpretation.

TRAFIC will follow the CONSORT reporting recommendations to describe a transparent statistical design reflecting relevant clinical opinion and decision making.

The re-purposing of drugs across diseases requires re-dosing and safety assessment and as the potential for repurposing increases, so does the potential to conduct trials with modelbased designs to more accurately estimate optimal dosing. The TRAFIC trial is the first reported dose finding trial of re-purposed treatment using a model-based design in inflammatory disease, conducted within the academic environment within research funding and regulatory constraints.

\section{TRIAL STATUS}

Protocol v11.00 21-March-2019. Open to recruitment: March-2015, recruitment completion expected June-2021.

\section{DECLARATIONS}

\section{Funding}

This research is supported by the MRC DPFS award MR/L005123/1. 


\section{Ethics Approval and Consent to Participate}

North East Ethics Approval Reference: 14/NE/1075.

All patients provide written informed consent to participate.

\section{Consent for Publication}

Not applicable.

No individual level details or images are included.

\section{Availability of Data and Materials}

Final trial dataset will be held by Newcastle University. Data sharing requests may be submitted following publication of results.

\section{Competing Interests}

The authors declare that they have no competing interests.

\section{Authors Contributions}

$\mathrm{MC}$ is trial statistician, developed the protocol and is author of the statistical analysis plan;

CY is statistical advisor; CB WN IM AF SS AP are clinical leads and developed the protocol;

JDI is Chief Investigator, conceived the trial, led the grant application and protocol

development; DDS is statistical co-applicant, led the study design and has protocol oversight. All authors have read and approved the final manuscript. 


\section{REFERENCES}

1. lasonos A, Wilton AS, Riedel ER, Seshan VE and Spriggs DR. A comprehensive comparison of the continual reassessment method to the standard $3+3$ dose escalation scheme in Phase I dose-finding studies. Clin Trials 2008;5:465-77

2. Wheeler GM, Mander AP, Bedding A, et al. How to design a dose-finding study using the continual reassessment method. Bmc Med Res Methodol 2019; 19: 18

3. Rogatko A, Schoeneck D, Jonas W, Tighiouart M, Khuri FR and Porter A. Translation of Innovative Designs into Phase 1 Trials. Clinical Oncology 2007;25(31):4982-6

4. van Brummelen EMJ, Huitema ADR, van Werkhoven E, Beijnen JH and Schellens JHM The performance of model-based versus rule-based phase I clinical trials in oncology. J Pharmacokinet Pharmacodyn 2016;43:235-42

5. N ational Audit Office. Services for people with rheumatoid arthritis. National Audit Office. 2009; 823: 1-37

6. Pickles T, Christensen R, Tam L-S, Simon LS and Choy EH. Early Phase and Adaptive Design Clinical Trials in Rheumatoid Arthritis: A systematic review of early phase trials. Rheumatology Advances in Practice 2018;2;1-11

7. McClue SJ, Blake D, Clarke $R$ et al. In vitro and in vivo anti-tumor properties of the cyclin-dependent kinase inhibitor CYC202 (R-roscovitine). Int J Cancer 2002;102:463-8

8. O'Quigley J, Pepe M, Fisher L. Continual reassessment method: a practical design for phase 1 clinical trials in cancer. Biometrics 1990;46(1),33-48

9. Cummins L, Katikireddi VS, Shankaranarayana S, Su KYC, Duggan E, Videm V, Pahau $\mathrm{H}$, Thomas R.Safety and retention of combination triple disease-modifying anti-rheumatic drugs in new onset rheumatoid arthritis. Intern Med J 2015;45(12):1266-73

10. Cheung YK. Dose finding by the continual reassessment method. Boca Raton (FL): CRC Press; 2011

11. Yap C, Billingham LJ, Cheung YK, Craddock C and O'Quigley J. Dose Transition Pathways: The Missing Link Between Complex Dose-Finding Designs and Simple Decision-Making. Clin Cancer Res 2017;23(24):7440-7

12. Yap C, Slade D, Brock K and Pan Y. dtpcrm: Dose Transition Pathways for Continual Reassessment Method. R package version 010. 2019

13. R Core Team. R: A Language and Environment for Statistical Computing. In: Computing RFfS, (ed.). Vienna, Austria 2017

14. Cheung K. dfcrm: Dose-finding by the continual reassessment method. $R$ package version 02-2. 2013 
15. A'Hern, R.P. Sample size tables for exact single-stage phase II designs. Statistics in Medicine 2001, 20, 859-866

16. Brown $S R$, Sherratt $D$, Booth $G$ et al. Experiences of establishing an academic early phase clinical trials unit. Clinical Trials 2017;14(4):349-356 
Table 1: Prior probability that each Dose Level is the MTD, $\delta=0.06$.

\begin{tabular}{|c|c|c|c|}
\hline \multirow[t]{2}{*}{ Dose level } & \multicolumn{3}{|c|}{ Prior SD of slope parameter $\beta, \sigma_{\beta}$} \\
\hline & 0.1 & $\sigma_{\beta}{ }^{L I}=0.265$ & $\sqrt{ } 1.34=1.16$ \\
\hline 1 (200mg) & 0.01 & 0.20 & 0.42 \\
\hline $2(400 \mathrm{mg})^{*}$ & 0.22 & 0.19 & 0.05 \\
\hline $3(600 \mathrm{mg})$ & 0.54 & 0.22 & 0.05 \\
\hline 4 (800mg) & 0.22 & 0.19 & 0.05 \\
\hline 5 (1000mg) & 0.01 & 0.20 & 0.42 \\
\hline
\end{tabular}

* Starting dose

Note: a large $\sigma_{\beta}$ does not necessary correspond to an uninformative prior for MTD level.

Table 2. Probability of DLT at each of five dose levels for five different dose-toxicity curves. Plateau calibration configuration curves for target DLT probability $\theta=0.35$ and five test dose levels.

\begin{tabular}{|c|c|c|c|c|c|}
\hline \multirow{2}{*}{ Curve } & \multicolumn{5}{|c|}{ Dose level } \\
\cline { 2 - 6 } & 1 & 2 & 3 & 4 & 5 \\
\hline 1 & $\mathbf{0 . 3 5}$ & 0.52 & 0.52 & 0.52 & 0.52 \\
\hline 2 & 0.21 & $\mathbf{0 . 3 5}$ & 0.52 & 0.52 & 0.52 \\
\hline 3 & 0.21 & 0.21 & $\mathbf{0 . 3 5}$ & 0.52 & 0.52 \\
\hline 4 & 0.21 & 0.21 & 0.21 & $\mathbf{0 . 3 5}$ & 0.52 \\
\hline 5 & 0.21 & 0.21 & 0.21 & 0.21 & $\mathbf{0 . 3 5}$ \\
\hline
\end{tabular}


Table 3. Summary statistics for the performance measures over the plateau calibration curves 2, 3, 4 and 5 for increasing levels of the indifference interval half-width $\delta$.

$\delta=0.06$ is highlighted; this is the level chosen for TRAFIC.

\begin{tabular}{|c|c|c|c|c|c|c|}
\hline$\delta$ & $\begin{array}{c}\text { Accuracy } \\
\text { Index } \\
\mathscr{A}_{N}\end{array}$ & $\begin{array}{c}\text { Probability } \\
\text { of correctly } \\
\text { selecting } \\
\text { MTD }\end{array}$ & $\begin{array}{c}\text { Mean } \\
\text { proportion } \\
\text { above MTD }\end{array}$ & $\begin{array}{c}\text { Mean } \\
\text { proportion } \\
\text { within } 1 \\
\text { dose of } \\
\text { MTD }\end{array}$ & $\begin{array}{l}\text { Mean } \\
\text { number } \\
\text { treated }\end{array}$ & SD $\mathscr{H}_{N}$ \\
\hline 0.02 & 0.46 & 0.51 & 0.17 & 0.79 & 19.3 & 0.12 \\
\hline 0.03 & 0.48 & 0.52 & 0.17 & 0.80 & 20.1 & 0.06 \\
\hline 0.04 & 0.46 & 0.51 & 0.17 & 0.79 & 20.3 & 0.05 \\
\hline 0.05 & 0.46 & 0.51 & 0.17 & 0.78 & 19.9 & 0.06 \\
\hline 0.06 & 0.46 & 0.50 & 0.16 & 0.78 & 19.7 & 0.06 \\
\hline 0.07 & 0.44 & 0.48 & 0.14 & 0.73 & 19.5 & 0.09 \\
\hline 0.08 & 0.43 & 0.48 & 0.13 & 0.73 & 19.4 & 0.11 \\
\hline 0.09 & 0.42 & 0.46 & 0.13 & 0.73 & 19.1 & 0.12 \\
\hline 0.10 & 0.41 & 0.45 & 0.11 & 0.71 & 18.9 & 0.14 \\
\hline 0.11 & 0.39 & 0.43 & 0.10 & 0.70 & 18.4 & 0.16 \\
\hline 0.12 & 0.38 & 0.42 & 0.10 & 0.70 & 18.3 & 0.17 \\
\hline 0.13 & 0.38 & 0.42 & 0.10 & 0.70 & 18.2 & 0.17 \\
\hline 0.14 & 0.37 & 0.41 & 0.10 & 0.69 & 17.7 & 0.18 \\
\hline 0.15 & 0.36 & 0.40 & 0.09 & 0.69 & 17.7 & 0.19 \\
\hline 0.16 & 0.36 & 0.40 & 0.09 & 0.69 & 17.6 & 0.19 \\
\hline 0.17 & 0.36 & 0.40 & 0.09 & 0.69 & 17.0 & 0.20 \\
\hline 0.18 & 0.36 & 0.39 & 0.09 & 0.69 & 17.0 & 0.21 \\
\hline 0.19 & 0.34 & 0.38 & 0.09 & 0.68 & 16.4 & 0.22 \\
\hline 0.20 & 0.32 & 0.36 & 0.08 & 0.66 & 15.8 & 0.21 \\
\hline
\end{tabular}


Table 4: Performance measures for simulated trials

\begin{tabular}{|c|c|c|c|c|c|c|c|c|c|c|}
\hline \multirow{3}{*}{$\begin{array}{c}\text { Simulation } \\
\text { Scenario }\end{array}$} & \multicolumn{5}{|c|}{ Assumed probability of DLT } & \multirow{3}{*}{$\begin{array}{c}\text { Accuracy } \\
\text { Index } \\
\mathscr{A}_{N}\end{array}$} & \multirow{3}{*}{$\begin{array}{l}\text { Probability of } \\
\text { correctly } \\
\text { selecting } \\
\text { MTD }\end{array}$} & \multirow{3}{*}{$\begin{array}{c}\text { Mean } \\
\text { proportion } \\
\text { above } \\
\text { MTD }\end{array}$} & \multirow{3}{*}{$\begin{array}{l}\text { Mean } \\
\text { proportion } \\
\text { within one } \\
\text { dose of } \\
\text { MTD }\end{array}$} & \multirow{3}{*}{$\begin{array}{l}\text { Mean } \\
\text { number } \\
\text { treated }\end{array}$} \\
\hline & \multicolumn{5}{|c|}{ Dose level } & & & & & \\
\hline & 1 & 2 & 3 & 4 & 5 & & & & & \\
\hline 1 & 0.14 & 0.23 & 0.35 & 0.47 & 0.57 & 0.91 & 0.50 & 0.22 & 0.95 & 19.7 \\
\hline 2 & 0.35 & 0.40 & 0.50 & 0.60 & 0.70 & 0.93 & 0.36 & 0.83 & 0.67 & 18.4 \\
\hline 3 & 0.15 & 0.35 & 0.40 & 0.50 & 0.60 & 0.92 & 0.45 & 0.46 & 0.88 & 19.3 \\
\hline 4 & 0.05 & 0.15 & 0.35 & 0.50 & 0.60 & 0.93 & 0.62 & 0.25 & 0.97 & 20.1 \\
\hline 5 & 0.05 & 0.15 & 0.25 & 0.35 & 0.60 & 0.92 & 0.50 & 0.08 & 0.78 & 20.0 \\
\hline 6 & 0.05 & 0.10 & 0.20 & 0.30 & 0.35 & 0.93 & 0.34 & 0.00 & 0.53 & 20.2 \\
\hline
\end{tabular}


Table 5: Final Trial Design Parameters

\begin{tabular}{|c|c|}
\hline Clinical Parameters & $\begin{array}{l}\text { DLT definition: cessation of IMP due to AE/ AR } \\
\text { Dose set: } 200,400,600,800,1000 \mathrm{mg} \text { daily } \\
\text { Starting dose: } 400 \mathrm{mg} \\
\text { Fixed or variable sample size: fixed, } n=21 \\
\text { Target level of DLT: } 35 \% \\
\text { Cohort size: } 3\end{array}$ \\
\hline $\begin{array}{l}\text { Model Specification } \\
\text { Parameters }\end{array}$ & $\begin{array}{l}\text { Model for dose-toxicity curve: Bayesian logistic } \\
\text { regression } \\
\text { Initial MTD prior: } 600 \mathrm{mg} \\
\text { One-stage or two-stage design: One } \\
\text { Estimation approach: Bayesian } \\
\text { Skeleton: }(0.14,0.23,0.35,0.47,0.57) \\
\text { Model: one parameter Bayesian model with prior } \\
\quad \text { distribution for slope } \mathrm{N}(\mu=0, \sigma=0.265)\end{array}$ \\
\hline Practical Considerations & $\begin{array}{l}\text { Skipping doses: No } \\
\text { Early stopping rules: Yes } \\
\text { i) High probability (>0.7) that posterior } \\
\text { probability of DLT at lowest dose is } \\
\text { greater than target DLT of } 35 \% \text {, indicating } \\
\text { that the lowest dose is too toxic } \\
\text { ii) } 4 \text { consecutive cohorts allocated MTD, } \\
\text { providing sufficient evidence MTD is } \\
\text { reached }\end{array}$ \\
\hline
\end{tabular}


Figure 1. Dose transition pathways flow diagram for first three cohorts

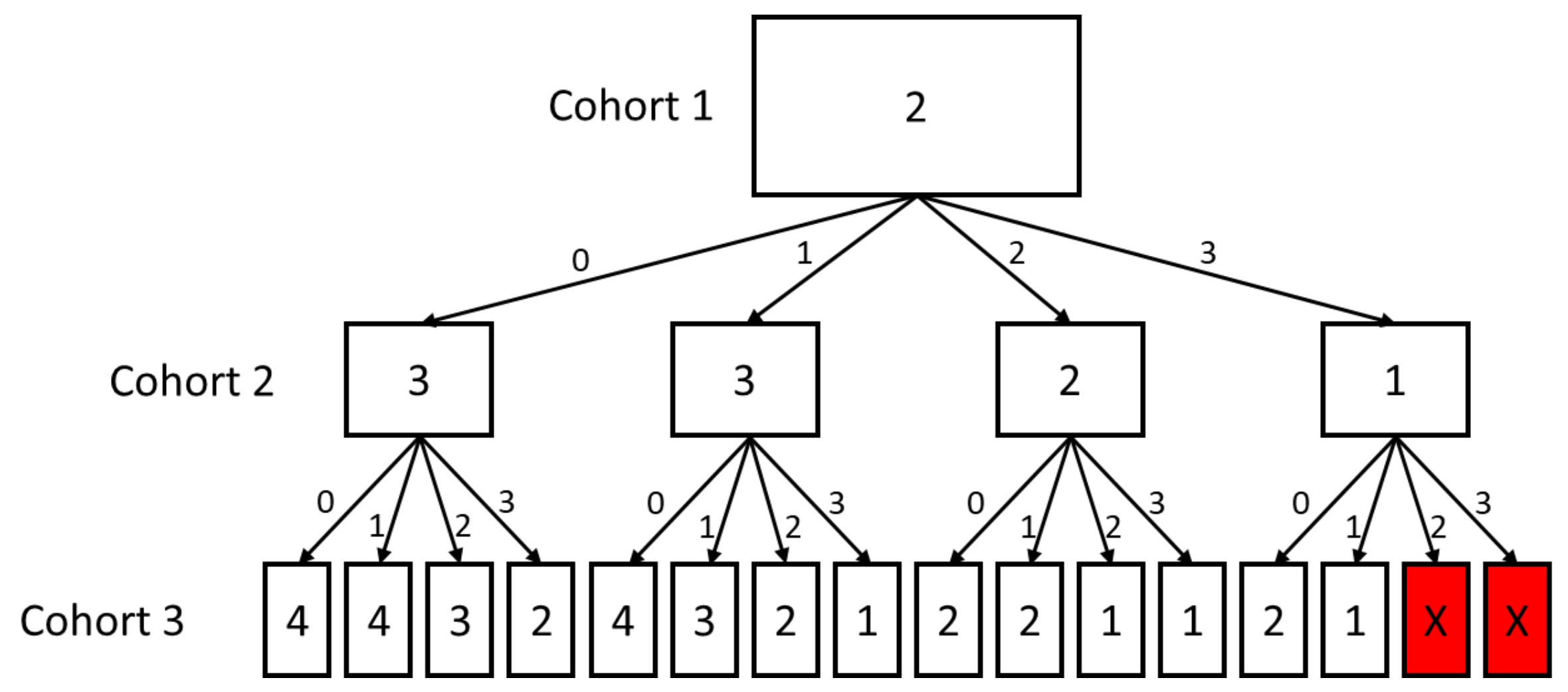

Numbers in boxes denote dose level of seliciclib: $1-200 \mathrm{mg} ; 2-400 \mathrm{mg} ; 3-600 \mathrm{mg} ; 4-800 \mathrm{mg}$. Numbers alongside arrows denote the number of DLTs in the preceding cohort. Boxes are shaded red when the recommendation is to stop the trial because the lowest dose is considered too toxic. 
Figure 2: Performance measures against the indifference interval half-width, $\delta$, for each of the plateau calibration curves. Early stopping rules are condition 1 and 2.

Early stopping rules are: Condition 1, Condition 2
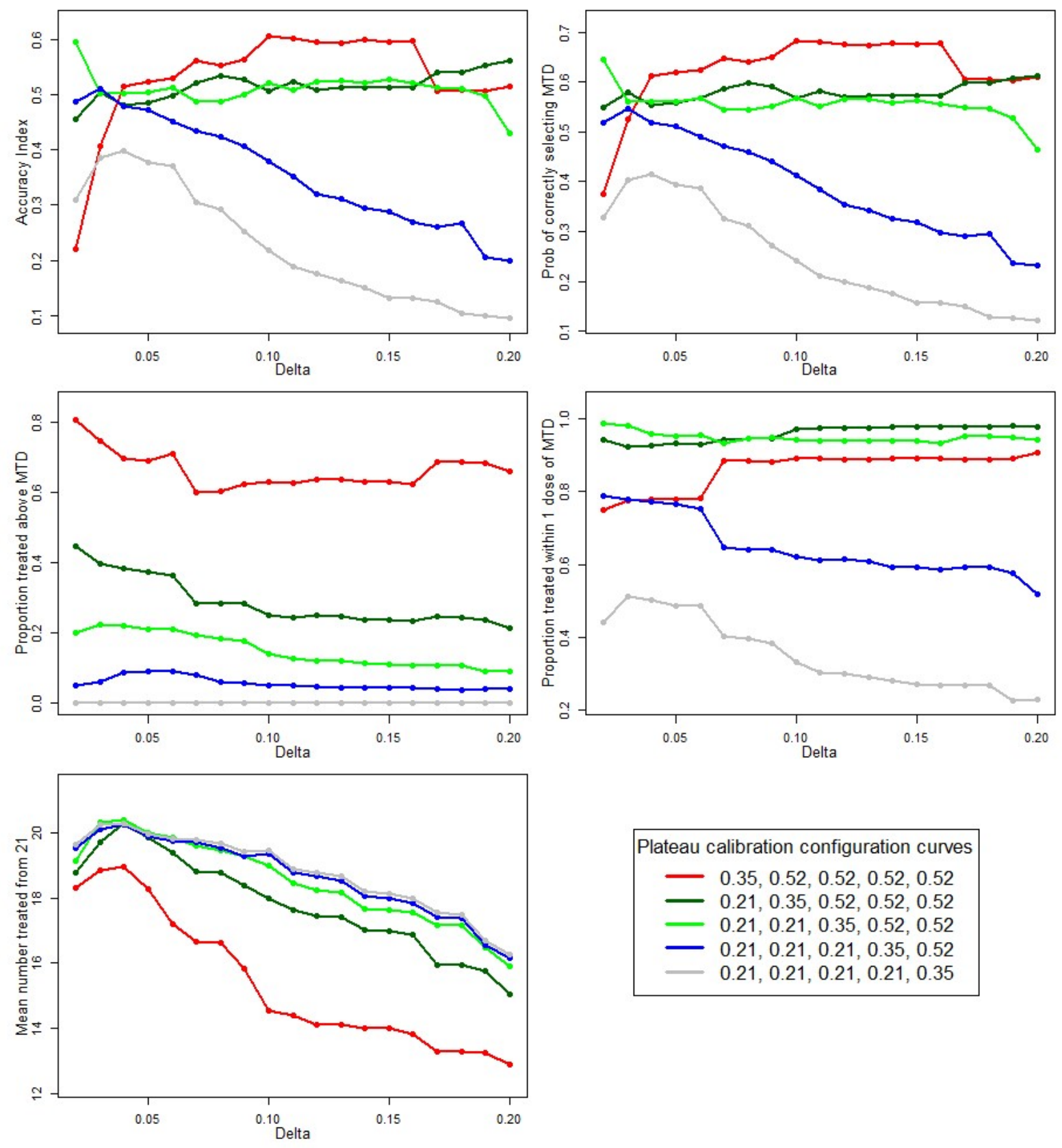

$$
\begin{gathered}
\text { Plateau calibration configuration curves } \\
-\quad 0.35,0.52,0.52,0.52,0.52 \\
-\quad 0.21,0.35,0.52,0.52,0.52 \\
-\quad 0.21,0.21,0.35,0.52,0.52 \\
-0.21,0.21,0.21,0.35,0.52 \\
-0.21,0.21,0.21,0.21,0.35
\end{gathered}
$$


Figure 3: Summary statistics for the performance measures taken over the plateau calibration curves 2, 3, 4 and 5. Performance measures are plotted against the indifference interval half-width Delta, $\delta$ and shown according to various stopping criteria

Summary measures taken over the plateau calibration configuration curves: 2, 3, 4, 5
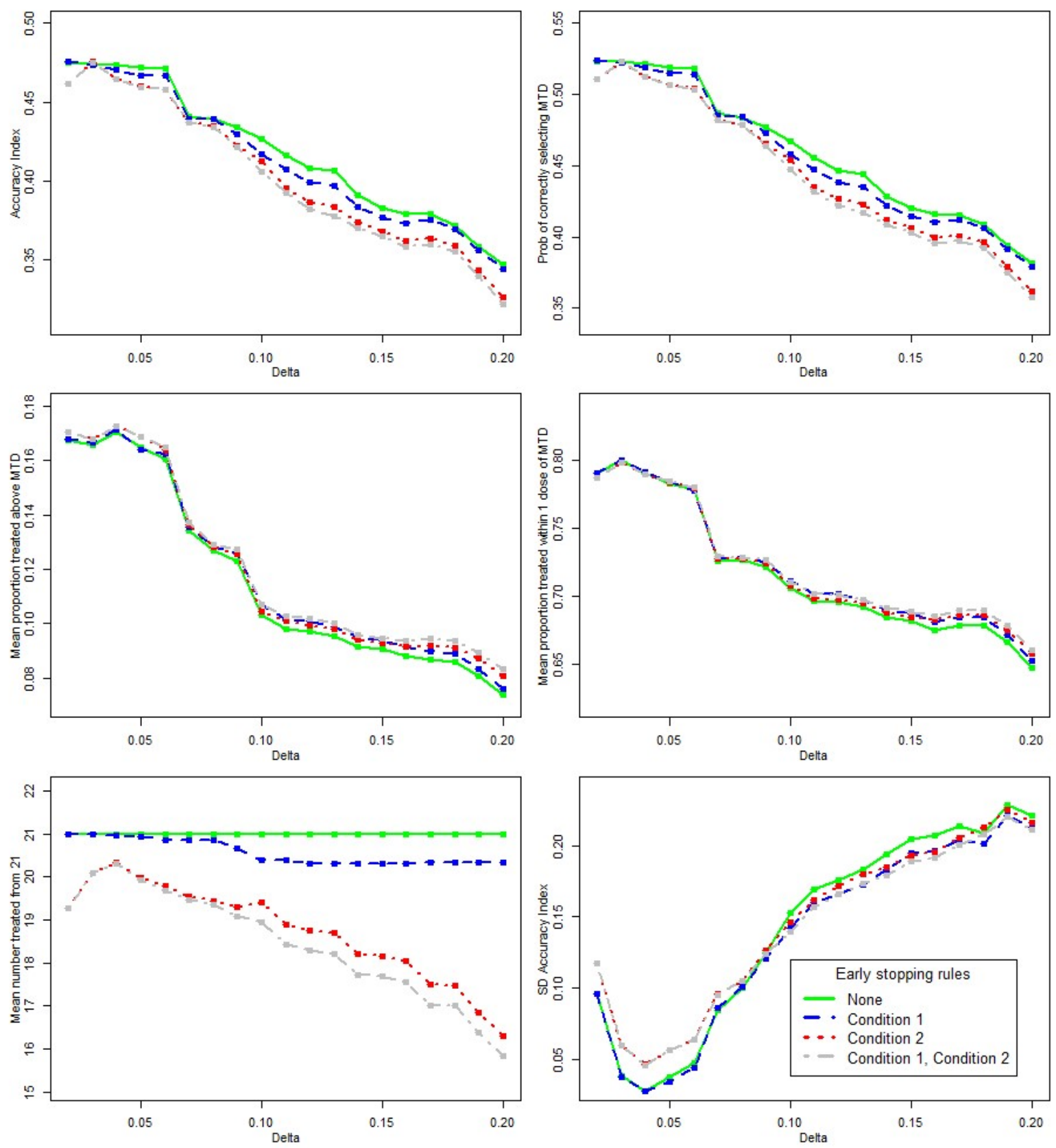


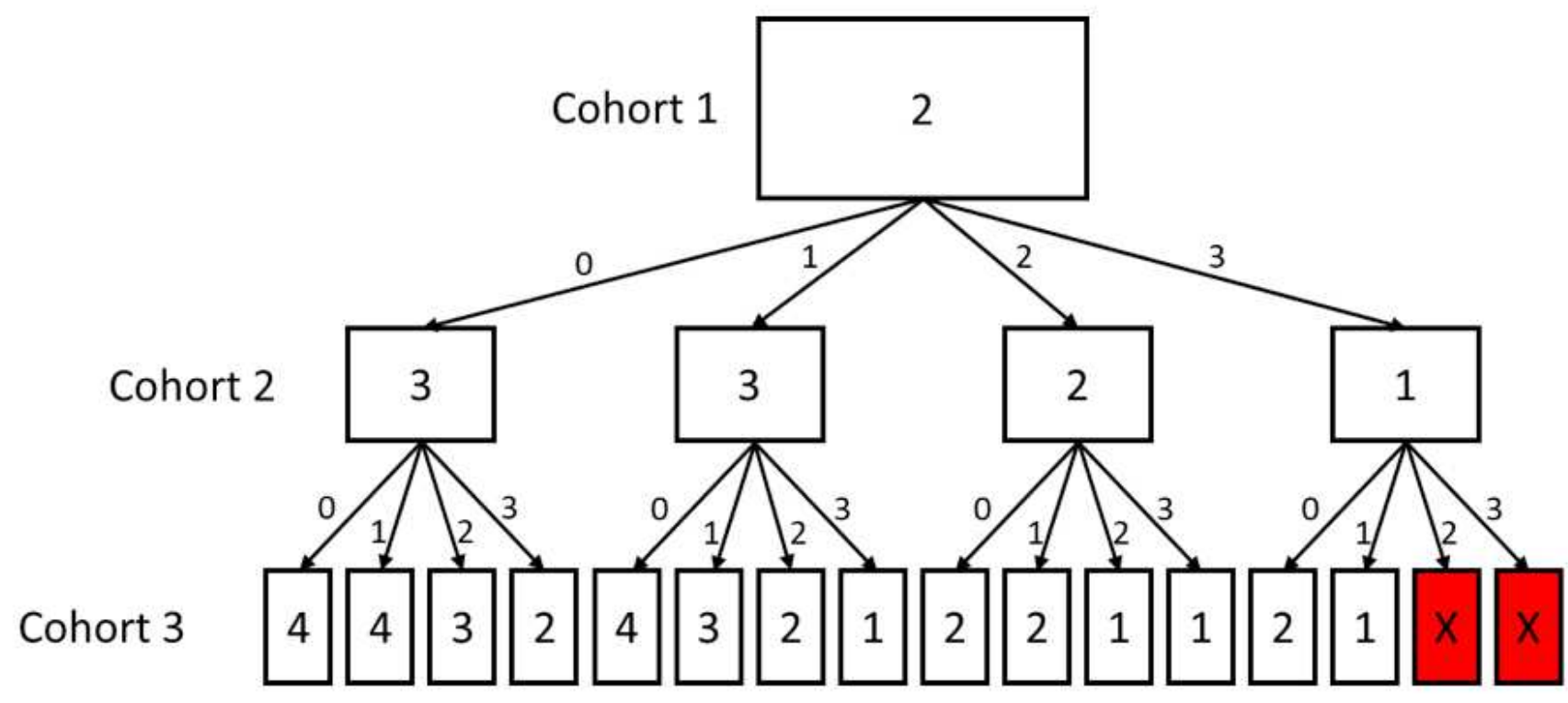

Numbers in boxes denote dose level of seliciclib: $1-200 \mathrm{mg} ; 2-400 \mathrm{mg} ; 3-600 \mathrm{mg} ; 4-800 \mathrm{mg}$. Numbers alongside arrows denote the number of DLTs in the preceding cohort. Boxes are shaded red when the recommendation is to stop the trial because the lowest dose is considered too toxic.

\section{Figure 1}

Dose transition pathways flow diagram for first three cohorts 
Early stopping rules are: Condition 1, Condition 2
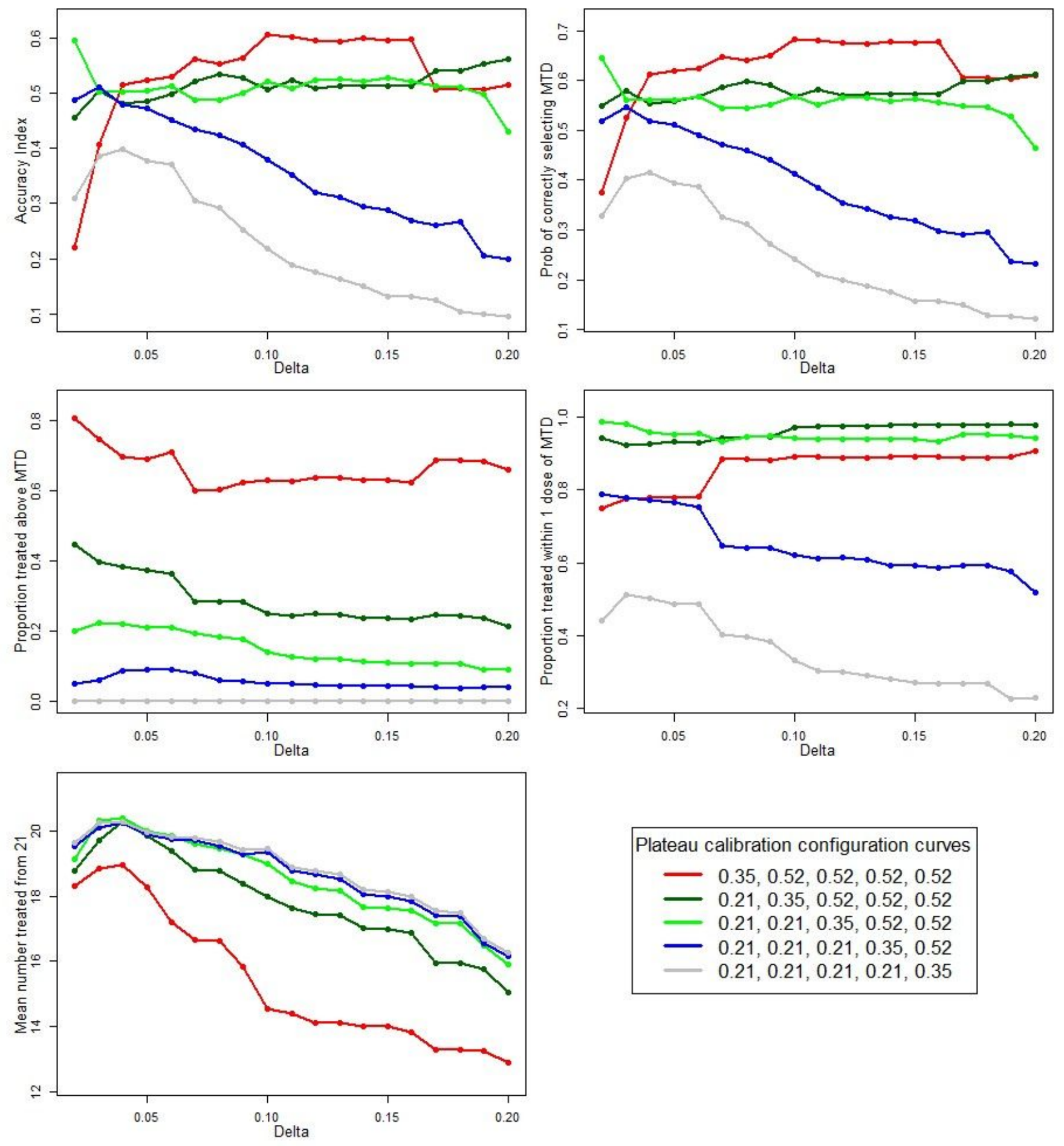

Figure 2

Performance measures against the indifference interval half-width, $\delta$ for each of the plateau calibration curves. Early stopping rules are condition 1 and 2. 
Summary measures taken over the plateau calibration configuration curves: 2, 3, 4, 5
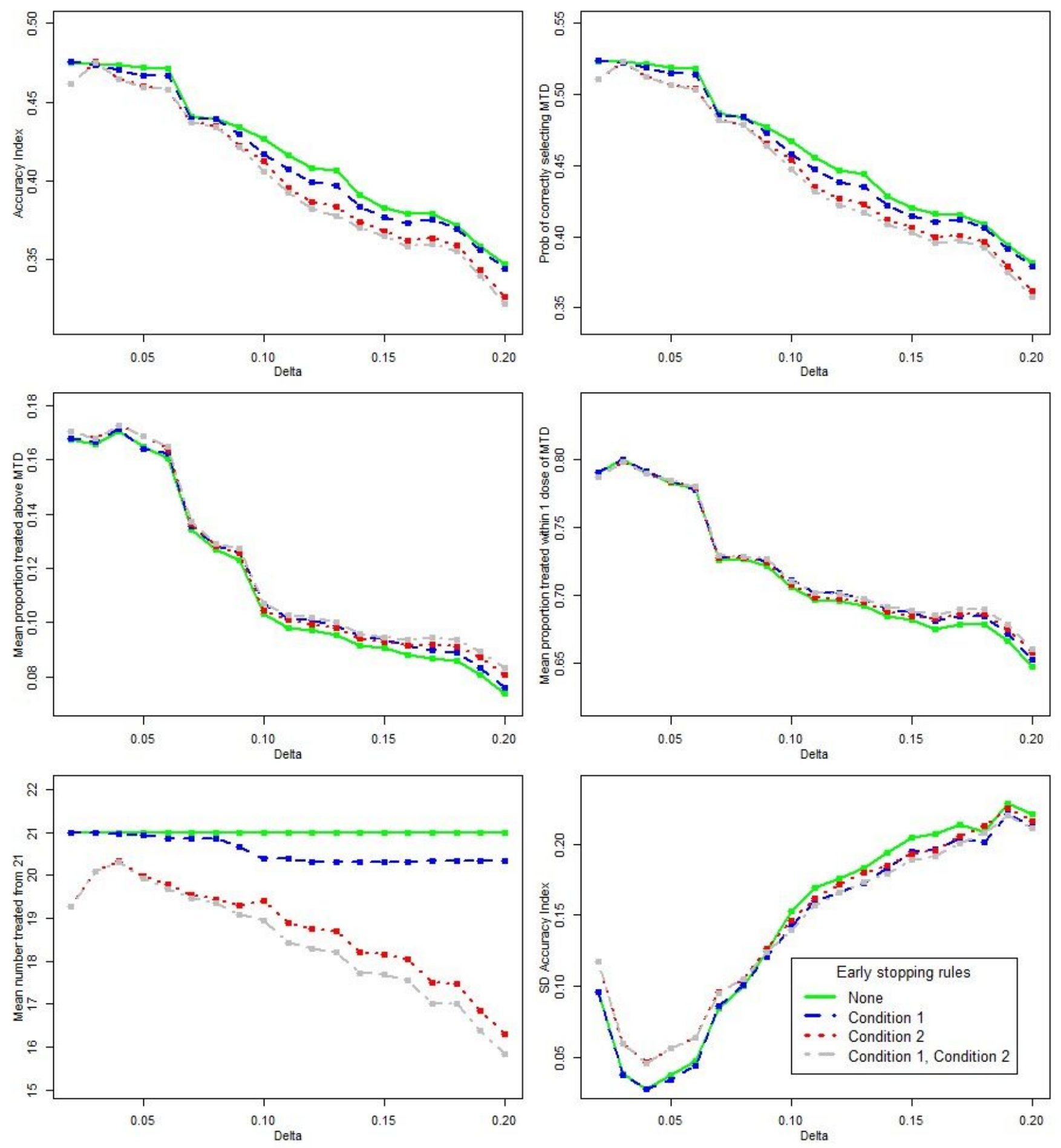

\section{Figure 3}

Summary statistics for the performance measures taken over the plateau calibration curves 2, 3, 4 and 5 . Performance measures are plotted against the indifference interval half-width Delta, $\delta$ and shown according to various stopping criteria 